\title{
Advancing Patient Care Through Specialty Pharmacy Services in an Academic Health System
}

\author{
Autumn Bagwell, PharmD, BCPS, AAHIVP; Tara Kelley, PharmD, CSP;
}

Alicia Carver, PharmD, BCPS, CSP; Jennifer B. Lee, PharmD; and Brandon Newman, PharmD, CSP

\begin{abstract}
BACKGROUND: With the rapid growth of specialty pharmacies, including those within academic health systems, pharmacists have the opportunity to improve patient care through the management of specialty medications. Specialty pharmacists within academic health systems are uniquely positioned to overcome restrictions to medication access, financial constraints, and provider burdens that often lead to obstacles for patients to start and maintain necessary treatments. The Vanderbilt Specialty Pharmacy (VSP) model at Vanderbilt University Medical Center (VUMC) provides an example of a patient-centered, collaborative care prototype that places pharmacists directly into specialty clinics to assist with comprehensive management of patients on specialty medications.
\end{abstract}

PROGRAM DESCRIPTION: VSP integrates specialty pharmacy services within existing specialty clinics based on the needs of each individual clinic. Each clinic is staffed with at least 1 clinical pharmacist and 1 pharmacy technician. The pharmacist is integrally involved in medication selection, initiation, and monitoring. The specialty pharmacy team ensures appropriate medication access and cost, provides extensive medication education, ensures patients are adherent to treatment, and coordinates care between patients and providers using the electronic medical record.

OBSERVATIONS: Integration of pharmacists within specialty clinics at VUMC benefits providers, the health system, and patient care. This model has demonstrated decreased provider and clinic burden, decreased time to medication approval and initiation, excellent patient and provider satisfaction, substantial patient cost savings, optimal medication adherence, and overall improved continuity of care for patients on specialty medications. Since its inception in 2011, VSP has integrated 24 clinical pharmacists and 17 pharmacy technicians into 20 specialty clinics, with continued quarterly growth.

IMPLICATIONS: The VSP model advances the role of pharmacists in managing patients on specialty medications in collaboration with providers. The integrated collaborative approach as presented by VSP represents a best practices model for those establishing and advancing specialty pharmacy services within academic health systems.

J Manag Care Spec Pharm. 2017;23(8):815-20

Copyright $\odot 2017$, Academy of Managed Care Pharmacy. All rights reserved.

\section{What is already known about this subject}

FDA approvals of specialty medications have grown exponentially over the last few years.

Health systems-based specialty pharmacies are emerging across the United States to improve access to specialty medications and better serve their patients.
Recent reports have described a variety of models for specialty pharmacies within health systems, but these reports have focused only on a few specialty disease states and have limited data demonstrating the benefits of these models.

\section{What this study adds}

This best practices report describes an inclusive model of integrated patient care for patients within an academic health system who are prescribed specialty medications.

Specific examples of basic and customizable specialty pharmacy services are described, which may be used in the development of comprehensive specialty medication management.

Improved patient outcomes are demonstrated, validating the success and benefits of this specialty pharmacy model.

$\bigcirc$ pecialty pharmacy is a rapidly growing field with significant potential to affect many health care sectors. Spending on specialty pharmaceuticals is predicted to increase to over $50 \%$ of total drug expenditures over the next 5 years. ${ }^{1}$ Because of expense and complexity, a keen understanding of each specialty medication is necessary. There has been a recent trend towards the development of specialty pharmacy services by health systems. The benefits of such a model encompass patients, providers, and the entire health system. This model improves communication between patients, providers, and pharmacists, and the time to medication approval and therapy initiation is decreased. ${ }^{2}$

Recent articles have discussed the involvement of pharmacists in a number of specialty clinics that focus on such specialty disease states as hepatitis $C$, human immunodeficiency virus, and multiple sclerosis. ${ }^{3-9}$ Within health systems, the design of specialty pharmacy practice models varies. Some systems combine existing ambulatory care services with new specialty pharmacy services, and other health systems establish new services where there were none previously.

The Vanderbilt Specialty Pharmacy (VSP) model at Vanderbilt University Medical Center (VUMC) provides patient-centered, collaborative care by placing pharmacists directly into specialty clinics to assist with comprehensive management of patients receiving specialty medications. This novel model has resulted in the rapid growth of specialty pharmacy services 
throughout the VUMC system and has improved patient care. This review describes the establishment and design of the VSP model and outcomes that highlight its value.

\section{Program Description}

\section{Establishing the VSP Services Model}

The integrated specialty pharmacy services model was initiated at VUMC in 2011. As of December 2016, VSP had a presence in 20 specialty clinics within VUMC and Monroe-Carell Jr. Children's Hospital. These clinics are staffed with 24 clinical pharmacists and 20 pharmacy technicians.

To establish the VSP integrated specialty pharmacy service model in any of the VUMC specialty clinics, a need is first recognized. Then, VSP leadership and clinical pharmacists meet with the specialty clinic directors and staff to discuss potential pharmacy services. Once onsite, the pharmacist begins to incorporate standard specialty services, while tailoring these services to the individual clinic (Table 1). Specialty pharmacists and pharmacy technicians function as the clinical extension of the VSP central pharmacy staff. As a relationship of trust develops between the clinic, providers, and VSP, the pharmacy team's role in the clinic evolves, addressing clinic needs in unique ways that decrease provider and nurse burden and advance the role of the pharmacist within the clinic.

Before the integration of VSP into the specialty clinics, the prior authorization (PA) and/or appeals process required by most insurance companies fell to an already overworked staff of nurses or other clinic staff, resulting in increased time to initiation of treatment. Patients were bewildered by the process and often abandoned therapy because of confusion, stress, or the high cost of obtaining medications. Often, the provider did not know that a patient was unable to access the prescribed therapy until the follow-up appointment, which could be months in the future. During this time, the patient's disease state could progress, leading to increased downstream medical costs and poor patient outcomes.

Integration of pharmacy teams into the clinics has streamlined medication selection and delivery and, most of all, approval. Once the decision is made to begin treatment, the pharmacy team is notified and the process begins (Figure 1). Providers within the clinics rely on the specialty pharmacists to evaluate whether prescribed treatments are the best option from an efficacy and safety standpoint, as well as the most costeffective regimen for patients. The time to initiating treatment is shortened significantly when a covered, affordable medication is initially prescribed.

PA paper work is completed by either a pharmacist or pharmacy technician, and if an appeal for coverage is required, the pharmacist uses his or her detailed knowledge of the medication and the disease state it treats to assist the provider in composing a letter that explains why the prescribed therapy was chosen for a particular patient. This process greatly reduces the

\section{TABLE 1 Vanderbilt Specialty Pharmacy Services}

\begin{tabular}{l|l}
\hline Basic VSP Services & Customizable Ancillary Services \\
\hline $\begin{array}{l}\text { Individualized initial and ongoing } \\
\text { patient counseling }\end{array}$ & $\begin{array}{l}\text { Pharmacist assistance with deter- } \\
\text { mining the most appropriate and } \\
\text { cost-effective treatment regimen }\end{array}$ \\
\hline $\begin{array}{l}\text { Insurance benefits investigation and } \\
\text { explanation }\end{array}$ & $\begin{array}{l}\text { Pretreatment immunization and lab } \\
\text { testing and imaging coordination }\end{array}$ \\
\hline Prior authorization/appeal assistance & $\begin{array}{l}\text { Ongoing lab monitoring and medi- } \\
\text { cation safety and efficacy follow-up }\end{array}$ \\
\hline Financial counseling & $\begin{array}{l}\text { Adherence information provided to } \\
\text { prescribers }\end{array}$ \\
\hline $\begin{array}{l}\text { Free shipping and delivery, free } \\
\text { ancillary supplies }\end{array}$ & $\begin{array}{l}\text { Collaborative practice agreements to } \\
\text { decrease provider burden }\end{array}$ \\
\hline Refill reminder calls & REMS program management \\
\hline $\begin{array}{l}\text { 24-hour access to on-call clinical } \\
\text { pharmacist }\end{array}$ & $\begin{array}{l}\text { Independent clinic visits with phar- } \\
\text { macist for treatment adherence and } \\
\text { side effects, efficacy, and drug inter- } \\
\text { action monitoring }\end{array}$ \\
\hline $\begin{array}{l}\text { Patient-centered individualized care } \\
\text { Continued monitoring of drug inter- } \\
\text { therapy modifications }\end{array}$ & \\
\hline
\end{tabular}

REMS = Risk Evaluation and Mitigation Strategy; VSP = Vanderbilt Specialty Pharmacy.

workload of clinic staff, primarily the nursing staff, while capitalizing on the pharmacist's strength in medication knowledge. Patients are thoroughly counseled, given time frames, updated frequently, and given a single point of contact who can answer their questions, which results in less stress for patients and better attitudes toward treatment.

After therapy is started, patients receive scheduled refill calls from pharmacy technicians associated with the clinic. If any issues arise during a standard set of questions asked during this call, the clinical pharmacist is notified to complete a patient assessment for adherence and medication safety and efficacy. If side effects are reported, the pharmacist can provide mitigating strategies and contact providers via the electronic medical record, thereby preventing abandonment of therapy and keeping providers informed of patients' treatment courses.

\section{VSP Integrated Services: The Infectious Diseases Clinic}

An excellent example of one of VSP's integrated clinics is the Infectious Diseases Clinic, where a clinical pharmacist is highly integrated in the management of patients with hepatitis $\mathrm{C}$ virus (HCV). In addition to the VSP pharmacy team's basic workflow outlined in Table 1 , the clinical pharmacist collaborates with providers in order to closely monitor patients on hepatitis $C$ treatment in the clinic and by telephone. The pharmacist is capable of ordering necessary labs, immunizations, and follow-up appointments to ensure that patients are monitored appropriately.

When a newly referred patient with HCV presents to the clinic, the pharmacist meets with that patient along with the provider to review possible treatment options and the approval 


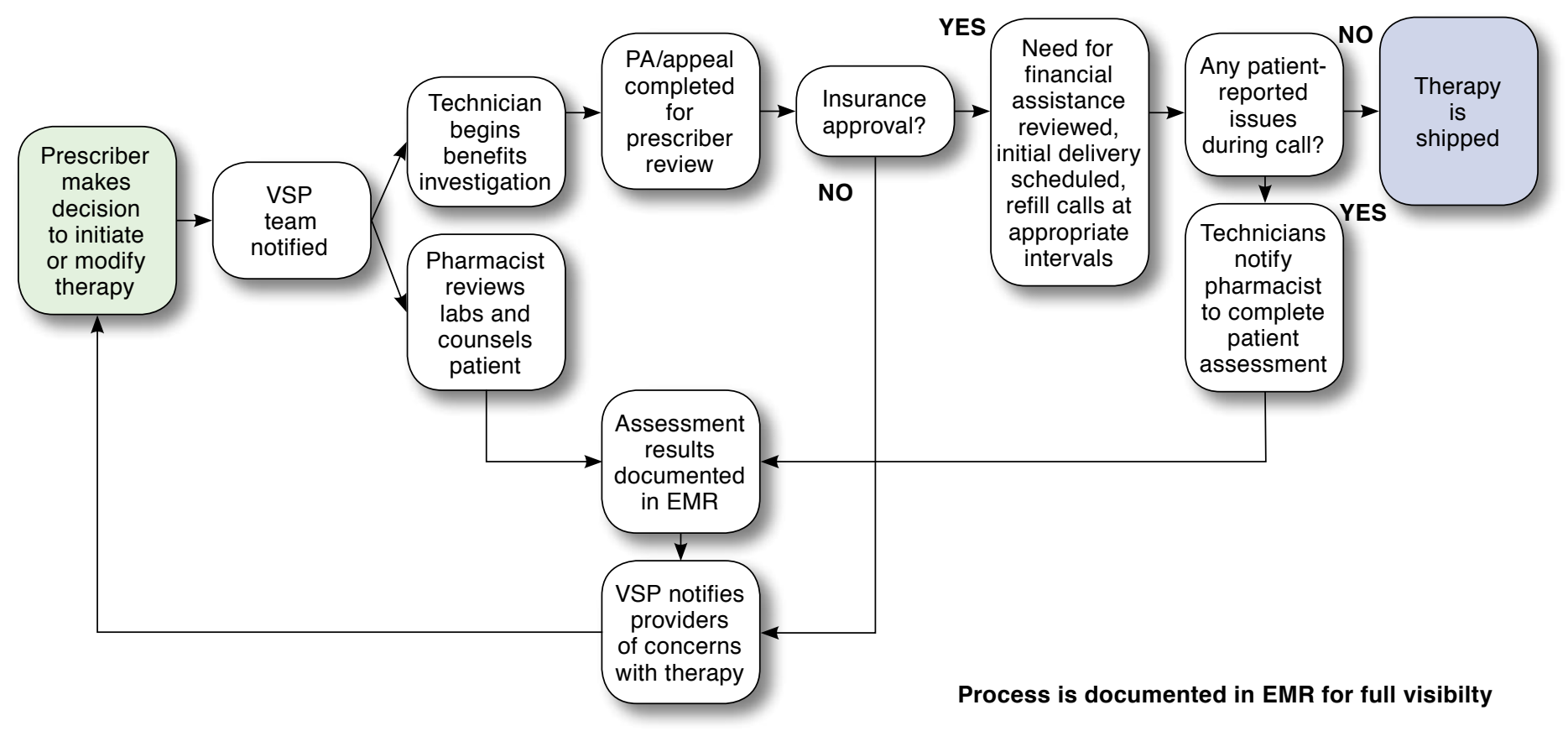

EMR=electronic medical record; $P A=$ prior authorization; VSP=Vanderbilt Specialty Pharmacy.

process with the available clinical information. The pharmacist completes a medication reconciliation, drug interaction evaluation, barriers to adherence assessment, and ensures that all appropriate laboratory testing and imaging are completed in a timely fashion. After medication is approved and financial assistance is obtained, the pharmacist educates the patient extensively by telephone, in the clinic, or by telecommunications video software. A follow-up visit is then arranged for the patient to be seen by the physician or pharmacist in the clinic for assessment and ongoing laboratory testing.

While the patient is receiving treatment, the pharmacist follows the patient closely with frequent telephone contact and independent clinic visits to ensure that the patient is adherent to laboratory testing schedules and medications and that there are no adverse effects or other concerns. If any side effects surface while on treatment, they can quickly be addressed and managed in order to maintain excellent adherence to the HCV regimen. As part of the patient's treatment, laboratory tests are also reviewed by the pharmacist and then discussed with the provider to determine if adjustments (such as dose modifications) are necessary.

\section{Diversity of Duties: The Multiple Sclerosis Center}

An example of the diversity of pharmacist duties in the specialty clinics is seen in the Vanderbilt Multiple Sclerosis Center, where 2 clinical pharmacists and 2 certified pharmacy technicians are integrated. As care coordinators for the self-adminis- tered multiple sclerosis (MS) specialty medications, the clinical pharmacists collaborate with other health care professionals, including physicians, nurses, social workers, and researchers to improve overall patient care. At the initiation of therapy, the pharmacists assess the appropriateness of therapy and ensure that all baseline testing has been completed and reviewed by the provider. The pharmacists provide extensive medication education to patients, clinic and pharmacy contact information, and expectations concerning the logistics of obtaining medication from a specialty pharmacy. This interaction is most often completed during a patient's clinic appointment with the patient's provider.

The pharmacists, in consultation with the MS providers, manage the PA and appeals processes in an effort to expedite the time to start treatment. With the help of the certified technicians, all PA documents are completed, including letters of medical necessity and letters of appeal, as well as coordination of any peer-to-peer reviews, if necessary. The pharmacists also work closely with clinical and administrative representatives from the respective pharmaceutical companies to ensure that patients have access to the appropriate support services and programs. Once treatment has been initiated, the pharmacists and pharmacy technicians perform regular patient assessments and are always available to patients and the clinic team members to manage adverse effects, promote treatment adherence, and encourage therapy adherence. 


\section{Observations}

\section{VSP Effect on Time to Treatment}

Within the Infectious Diseases Clinic, the integrated process allows the pharmacist to see patients for follow-up visits to ensure work-up completion, which allows providers additional open clinic appointments to see new patients, thereby increasing the number of patients able to obtain treatment. The quarter before VSP's transition to the integrated model was compared with the median of the subsequent 3 quarters after transition. Benefits to providers and the health system were evidenced by a $34 \%$ decrease in pretreatment appointments ( 1.14 down to 0.75 clinic visits per day); an $80 \%$ decrease in on-treatment provider appointments, since these were seen by the pharmacist ( 0.36 down to 0.1 clinic visits per day); and a $38 \%$ increase in prescriptions for $\mathrm{HCV}$ treatment generated from the clinic (0.8 up to 1.1 prescriptions generated per clinic day). Patient benefit was also shown, with a $78 \%$ decrease in time to medication approval (median of 67 days down to 15 days) and a $68 \%$ decrease in time to medication initiation (median of 82 days down to 26 days) from an initial clinic visit.

\section{VSP Effect on Access to Medication}

In addition to the Infectious Diseases Clinic, many patients with hepatitis $C$ are treated in the VUMC Digestive Disease Center, where 2 clinical pharmacists and 1 certified pharmacy technician are integrated. The positive effect of the VSP presence within this clinic is evident in the exceptional rate of patient access to medication. After an initial PA request, only $75 \%$ of prescriptions for these medications were approved by commercial and federal pharmacy benefit managers, leaving $25 \%$ of these patients without initial access to medication. However, VSP pharmacists were able to provide medication access to $100 \%$ of these patients who were initially denied treatment through their pharmacy benefit managers. This was achieved through either insurance appeals (97\%) or enrollment into manufacturer assistance programs (3\%), both of which were completed by VSP pharmacists. This level of medication access far surpasses recent data reported by a large pharmacy network showing a non-start rate of $45 \%$ in patients with commercial insurance. ${ }^{10}$

\section{VSP Effect on Patient-Centered Care}

The positive effect of the VSP pharmacy team on patient-centered individualized care is further demonstrated in the HCV treatment completion rate of $97 \%$. The majority of patients who filled HCV medications through VSP had no copay (53\%). While not assessed by this study, this high level of completion of HCV medications compared with other studies that looked at real-world HCV treatment completion is likely correlated to the integrated services provided in this clinic. ${ }^{11-13} \mathrm{HCV}$ pharmacists ensure cost-effective access to treatment and the necessary support for patients to maintain that treatment.
The Lipid Clinic, which has more recently integrated VSP services, also exemplifies the positive effect of VSP on patient care. Specialty medications prescribed in this clinic include PSCK9 inhibitors, which have been difficult for patients to access because of insurance denials. Since the integration of VSP into this clinic, over $95 \%$ of patients who met the criteria for this medication have been able to gain approval for these medications and begin treatment. VSP hopes to expand this type of integrative care into more specialty clinics to further develop the pharmacist's role in patient care.

\section{VSP Effect on Patient Adherence}

Medication effectiveness is often associated with patient adherence; therefore, payers often use quality measures to evaluate the effectiveness of a specialty pharmacy to maintain adherence to a medication regimen. ${ }^{14,15}$ Medication possession ratio (MPR) is one method used to evaluate the adherence of patients to their specialty medications. Many payers require at least an $80 \%$ MPR rate for chronic inflammatory diseases, since this is commonly regarded as the definition for medication adherence ${ }^{16}$ For the fiscal year (FY) 2016, VSP exceeded this minimum rate with an overall monthly MPR above $96 \%$, which comprised all 20 clinics (over 4,000 patients) that had a clinical pharmacist integrated into their workflow.

Patients' satisfaction with their specialty pharmacies can also be correlated to medication adherence. At VSP, patient satisfaction is evaluated by quarterly surveys sent to all patients $(>4,000)$ of VSP clinics. Patients rate various points of contact with their specialty pharmacies, as well as their overall experience. The overall experience is rated on a scale of 1 to 5 , with 1 being "poor" and 5 being "great." Notably, the most recent quarterly evaluation report included feedback from 1,448 unique patients ( $36 \%$ response rate) who rated VSP at an average of 4.93 out of 5 . VSP attributes these high satisfaction ratings to the personalized experience each patient receives through this unique service.

\section{VSP and Financial Assistance}

Improved adherence and overall patient satisfaction is to be expected because of VSP's commitment to patients. Often, cost is a barrier to access for patients who are prescribed specialty medications. Research suggests that barriers exist when prescription cost is beyond $\$ 100$, concluding that increased out-ofpocket cost decreases adherence rates. ${ }^{17,18}$ The Pharmacy Benefit Management Institute reports that the average patient out-ofpocket expense for specialty medications from 2014 to 2015 was $\$ 76 . .^{19}$ VSP ensures that patients have every opportunity to receive their specialty medications, even if they have difficulty affording them. Many medications are dispensed at no cost to patients, preventing cost from being a barrier to adherence.

Manufacturers, as well as nonprofit organizations, offer many resources for patients who have difficulty affording their 
medications. VSP assists these patients in finding the most appropriate resource for their specific needs, including various free drug programs directly through manufacturer assistance or free drug programs and copay cards that are handled and dispensed through VSP. In FY 2016, VSP assisted patients in saving $\$ 5,811,640.92$ through various copay or other patient assistance programs, helping to dispense 14,068 prescriptions that may not otherwise have been obtained. This figure does not include the amount of assistance VSP has helped patients to receive through the direct-from-manufacturer programs or the e-vouchers that are automatically applied to some commercial insurance plans.

VUMC, a nonprofit organization, also offers patient assistance through a Medication Access Program Grant. Patients who express an inability to afford their medications and are unable to qualify for assistance through other programs are evaluated for this grant, which is funded by VUMC. For FY 2016 , specialty patients received $\$ 2,110,165$ in assistance through this grant, allowing for over 4,500 prescriptions to be filled for patients that may have otherwise been unable to afford their treatment.

VSP patients were able to receive $\$ 7,911,805.92$ in financial aid with the guidance of VSP's financial assistance team. Based on FY 2016, VSP patients most often paid nothing out of pocket for their prescriptions (mode $\$ 0$ ). On average, a patient's out-of-pocket expense is $\$ 36.20$ per prescription, with typical spending of $\$ 8.76$ for prescriptions (median). This amount is $88.5 \%$ lower than the average out-of-pocket cost reported by the Pharmacy Benefit Management Institute. VSP continues to strive to improve patient outcomes through patient adherence.

\section{Implications}

Disease states requiring specialty medications are often confusing, burdensome, and overwhelming for patients. The goal of VSP is to make obtaining these specialty medications as simple and stress-free as possible. The integrated pharmacy services model is innovative and beneficial to all parties involved. The VUMC health system benefits from the influx of specialty medication prescriptions back into the Vanderbilt system. The providers benefit from the specialized knowledge provided by the pharmacists and the improved patient access to medications. The clinic staff is relieved of the burden of obtaining and maintaining insurance authorization, as well as keeping up with monitoring requirements, which allows them more time to focus on other aspects of patient care. Most important, patients benefit by developing trusted relationships with their designated pharmacy teams, who provide support systems to empower them during therapy.

Ensuring affordable medication costs and providing enhanced monitoring while on therapy ultimately leads to superior patient outcomes, which is the overall goal for VSP. Further research on the role of specialty pharmacists in decreasing overall health care costs, additional specific diseasestate interventions pharmacists may provide when integrated in clinics, and disease state-specific medication access restrictions is warranted and may be beneficial in future descriptions of best practices in specialty pharmacy.

\section{Authors}

AUTUMN BAGWELL, PharmD, BCPS, AAHIVP; TARA KELLEY, PharmD, CSP; ALICIA CARVER, PharmD, BCPS, CSP; JENNIFER B. LEE, PharmD; and BRANDON NEWMAN, PharmD, CSP, Vanderbilt Specialty Pharmacy, Nashville, Tennessee.

AUTHOR CORRESPONDENCE: Autumn Bagwell, PharmD, BCPS, AAHIVP, Vanderbilt Specialty Pharmacy, 1301 Medical Center Dr., Nashville, TN 37232. Tel.: 615.936.6353; E-mail: Autumn.D.Bagwell@vanderbilt.edu.

\section{DISCLOSURES}

No outside funding supported this study. The authors have nothing to disclose.

Study concept and design were principally contributed by Bagwell and Newman, along with the other authors. Lee took the lead in data collection, along with Carver, Bagwell, Kelley, and Newman. Data interpretation was performed by Carver, Kelley, Lee, and Bagwell, with assistance from Newman. The manuscript was written by Bagwell, Carver, Kelley, and Lee and revised primarily by Bagwell, along with the other authors.

\section{REFERENCES}

1. Colgan K, Beacher R. Importance of specialty pharmacy to your health system. Am J Health Syst Pharm. 2015;72(9):753-56.

2. Hanson RL. Specialty pharmacy and the medication access dilemma Am J Health Syst Pharm. 2015;72(9):693.

3. Hanson RL, Habibi M, Khamo N, Abdou S, Stubbings J. Integrated clinical and specialty pharmacy practice model for management of patients with multiple sclerosis. Am J Health Syst Pharm. 2014;71(6):463-69.

4. Habibi M, Kuttab HM. Management of multiple sclerosis and the integration of related specialty pharmacy programs within health systems. Am J Health Syst Pharm. 2016;73(11):811-19.

5. Fajardo S, Zook F, Dotson E. Specialty pharmacy for hematologic malignancies. Am J Health Syst Pharm. 2016;73(11):797-809.

6. Gilbert EM, Gerzenshtein L. Integration of outpatient infectious diseases clinic pharmacy services and specialty pharmacy services for patients with HIV infection. Am J Health Syst Pharm. 2016;73(11):757-63.

7. Mullican KA, Francart SJ. The role of specialty pharmacy drugs in the management of inflammatory diseases. Am J Health Syst Pharm. 2016;73(11):821-30.

8. Rim MH, Smith L, Kelly M. Implementation of a patient-focused specialty pharmacy program in an academic healthcare system. Am J Health Syst Pharm. 2016;73(11):831-38.

9. Khiani V, Stubbings J. The pharmacist's role in biologic management for IBD in a health system-integrated practice model. Am J Pharm Benefits. 2015;7(5):215-20

10. Younossi ZM, Bacon BR, Dieterich DT, et al. Disparate access to treatment regimens in chronic hepatitis $C$ patients: data from the TRIO network. J Viral Hepat. 2016;23(6):447-54. 
11. Backus LI, Belperio PS, Shahoumian TA, Loomis TP, Mole LA. Effectiveness of sofosbuvir-based regimens in genotype 1 and 2 hepatitis $C$ virus infection in 4026 U.S. veterans. Aliment Pharmacol Ther. 2015;42(5):559-73.

12. Tsai N, Bacon BB, Flamm S, et al. Comparison of sofosbuvir \pm simeprevir in heterogeneous, real-world populations of $\mathrm{HCV}$ patients over 70 years: data from the TRIO network. Gastroenterology. 2015;148(4 Suppl 1):S-1085 [Abstract Tul017]. Available at: http://www.gastrojournal.org/article/S00165085(15)33706-9/pdf. Accessed June 24, 2017.

13. Kowdley K, Bacon BR, Dieterich D, et al. Efficacy evaluation of 24 week $\mathrm{SOF}+\mathrm{RBV}$ in a heterogeneous, real-world population of genotype $3 \mathrm{HCV}$; data from the TRIO network. Gastroenterology. 2015;148(4 Suppl 1):S-1087 [Abstract Tu1023]. Available at: http://www.gastrojournal.org/article/S00165085(15)33711-2/abstract. Accessed June 24, 2017.

14. Agency for Healthcare Research and Quality. Medication adherence interventions: comparative effectiveness. Closing the quality gap: revisiting the state of the science. Executive summary. Evidence Report No. 208 (AHRQ Publication No. 12-E010-1). 2012. Available at: http://www.effectivehealthcare ahrq.gov/ehc/products/296/1249/EvidenceReport208_CQGMedAdherence_ ExecutiveSummary_20120904.pdf. Accessed June 24, 2017.
15. Stefanacci RG, Guerin S. Why medication adherence matters to patients, payers, providers. Manag Care. 2013;22(1):37-39.

16. Blue Cross Blue Shield Association Technology Evaluation Center. Medication adherence. TEC Special Report. Volume 29, no. 13. March 2015. Blue Cross Blue Shield Association.

17. Streeter SB, Schwartzberg L, Husain N, Johnsrud M. Patient and plan characteristics affecting abandonment of oral oncolytic prescriptions. J Oncol Pract. 2011;7(3 Suppl):46s-51s.

18. Goldman DP, Joyce GF, Zheng Y. Prescription drug cost sharing: associations with medication and medical utilization and spending and health. JAMA. 2007;298(1):61-69.

19. Pharmacy Benefit Management Institute. PBMI Research report: trends in specialty drug research. Walgreens Specialty Pharmacy. 2016. Available at: https://www.pbmi.com/ItemDetail?iProductCode=Specialty_2016\&Categ ory=SPECIALTY. Accessed June 24, 2017. 